July 1995

Imperial/TP/94-95/50

\title{
Unitary Theory of Evaporating 2D Black Holes
}

\author{
Aleksandar Miković 曰 \\ Blackett Laboratory, Imperial College, Prince Consort Road, London SW7 2BZ, UK \\ and \\ Institute of Physics, P.O. Box 57, 11001 Belgrade, Yugoslavia ? \\ E-mail: mikovic@castor.phy.bg.ac.yu
}

\begin{abstract}
We study a manifestly unitary formulation of $2 \mathrm{~d}$ dilaton quantum gravity based on the reduced phase space quantization. The spacetime metric operator can be expanded in a formal power series of the matter energymomentum tensor operator. This expansion can be used for calculating the quantum corrections to the classical black hole metric by evaluating the expectation value of the metric operator in an appropriate class of the physical states. When the normal ordering in the metric operator is chosen to be with respect to Kruskal vacuum, the lowest order semiclassical metric is exactly the one-loop effective action metric discovered by Bose, Parker and Peleg. The corresponding semiclassical geometry describes an evaporating black hole which ends up as a remnant. The calculation of higher order corrections and implications for the black hole fate are discussed.
\end{abstract}

\footnotetext{
${ }^{1}$ Work supported by MNTRS and Royal Society

${ }^{2}$ Permanent address
} 


\section{Introduction}

Hawking's discovery that black holes radiate thermally [1], gave rise to a series of questions about the quantum fate of a black hole. Is the quantum evolution of an evaporating black hole unitary or not? Related to that are the questions of information loss and the final spacetime geometry. A proper framework for answering these questions would be a quantum theory of gravity, but in the absence of such a theory the best one can do is to study toy models of black hole formation and evaporation.

Two-dimensional dilaton gravity models coupled to matter (CGHS and its modifications [2, 3, 15]) represent such toy models, and they have been extensively studied due to their classical and quantum solvabilty. One aspect of the quantum solvability is the fact that the exact canonical constrained quantization can be performed for $N=24$ matter fields and the physical Hilbert space can be obtained, which is the free-field matter Fock space [4, 5, 6, 7]. Furthermore, it has been noticed that the constraints can be deparametrized, or equivalently a time variable can be found [6, 7], which allows for a construction of a unitary quantum evolution operator. This can be done explicitely in the reduced phase space formalism (i.e. by fixing a gauge and solving the constraints in terms of independent canonical variables), and a unitary quantum theory can be constructed [8]. The metric operator in the Heisenberg picture can be expressed as an operator valued solution of the classical equations of motion, and its expectation value in a physical state determines an effective quantum metric.

Calculating the quantum corrections and backreaction turns out to be easier in this unitary gauge formalism then in the effective action approach. One expands the metric operator into a formal power series in the matter energy-momentum tensor operator and takes the expectation value in the appropriate physical state [8]. By choosing a normal ordering in the metric operator to be with respect to the "out" vacuum corresponding to the background of the classical black hole solution, and by taking the initial state to be a coherent state with respect to the "in" dilaton vacuum, the lowest order semiclassical metric was obtained. It was the black hole metric plus the back-reaction correction due to the Hawking radiation. This metric was different from the other one-loop semiclassical metrics obtained in the effective action approach, and it was conjectured that different normal orderings in the unitary gauge formalism correspond to different combinations of the one-loop counterterms in the effective action.

In this paper we show that a normal ordering in the metric operator with respect to Kruskal vacuum gives the lowest order semiclassical metric which is the same as 
the semiclassical metric obtained by Bose, Parker and Peleg (BPP) from a one-loop effective action [9]. In section 2 we describe the reduced phase space formalism for the CGHS model. In section 3 we quantize the reduced phase space formulation, and define the quantum metric and the class of relevant physical states. In section 4 we show how the BPP solution arises in the semiclassical limit of our quantum theory. In section 5 we describe how to calculate the second order quantum corrections, and in section 6 we present our conclussions.

\section{Reduced phase space formalism}

In the reduced phase space formalism one solves the constraints classicaly and then quantize the independent set of canonical variables. Solving the constraints requires a gauge fixing, and one has to take care that a good gauge is chosen. The advantage of this method is that one can obtain the physical Hilbert space relatively easy. The disadvantage is that the gauge symmetries (which are generated by the constraints) are not manifest. In the case of black hole toy models, this strategy was used for analysing the spherically symmetric black hole collaps (BCMN model) 10, 11, 12], which can be recasted in the $2 \mathrm{~d}$ dilaton gravity form [3]. However, the problem there is that the BCMN gauge does not penetrate the horizon, and consequently the Hamiltonian evolution stops at the horizon [12]. Also the Hamiltonian of the BCMN model is a non-local function of the matter fields, so it is difficult to promote it into a Hermitian operator. In the case of the CGHS model such problems are absent [8], and the reduced phase space approach can be implemented.

We start from the classical CGHS action [2]

$$
S=\int_{M} d^{2} x \sqrt{-g}\left[e^{-\phi}\left(R+(\nabla \phi)^{2}+4 \lambda^{2}\right)-\frac{1}{2} \sum_{i=1}^{N}\left(\nabla f_{i}\right)^{2}\right]
$$

where $\phi$ is a dilaton, $f_{i}$ are scalar matter fields, $g, R$ and $\nabla$ are determinant, scalar curvature and covariant derivative respectively, associated with a metric $g_{\mu \nu}$ on the $2 \mathrm{~d}$ manifold $M$. Topology of $M$ is that of $\mathbf{R} \times \mathbf{R}$. We make a field redefinition

$$
\tilde{g}_{\mu \nu}=e^{-\phi} g_{\mu \nu} \quad, \quad \tilde{\phi}=e^{-\phi}
$$

so that one obtains a simpler action

$$
S=\int_{M} d^{2} x \sqrt{-\tilde{g}}\left[\tilde{R} \tilde{\phi}+4 \lambda^{2}-\frac{1}{2} \sum_{i=1}^{N}\left(\tilde{\nabla} f_{i}\right)^{2}\right] .
$$

Canonical analysis of the action (2.3) gives

$$
S=\int d t d x\left(\pi_{\tilde{\rho}} \dot{\tilde{\rho}}+\pi_{\tilde{\phi}} \dot{\tilde{\phi}}+\pi_{f} \dot{f}-N_{0} G_{0}-N_{1} G_{1}\right)-\int d t H
$$


where $N_{0}$ and $N_{1}$ are the rescaled laps and shift, while the Hamiltonian constraint $G_{0}$ and the spatial diffeomorphisms constraint $G_{1}$ are given as

$$
\begin{aligned}
& G_{0}=-\pi_{\tilde{\rho}} \pi_{\tilde{\phi}}-4 \lambda^{2} e^{\tilde{\rho}}+2 \tilde{\phi}^{\prime \prime}-\tilde{\rho}^{\prime} \tilde{\phi}^{\prime}+\frac{1}{2}\left(\pi_{f}^{2}+\left(f^{\prime}\right)^{2}\right) \\
& G_{1}=\pi_{\tilde{\phi}} \tilde{\phi}^{\prime}+\pi_{\tilde{\rho}} \tilde{\rho}^{\prime}-2 \pi_{\tilde{\rho}}^{\prime}+\pi_{f} f^{\prime} .
\end{aligned}
$$

The primes stand for the $x$ derivatives, $\tilde{\rho}$ is the conformal factor $\left(\tilde{g}=e^{\tilde{\rho}}\right)$, and we have taken a single matter field $(N=1)$ for the simplycity sake. The boundary term in (2.4) is needed in order to obtain correct equations of motion [13], and $H$ will be the Hamiltonian in the physical gauge.

Now we fix the gauge

$$
\tilde{\rho}=0 \quad, \quad \pi_{\tilde{\phi}}=0,
$$

which can be thoutght of as the $2 \mathrm{~d}$ dilaton gravity analog of the BCMN gauge [10]. However, unlike the BCMN gauge, (2.6) penetrates the horizon and corresponds to the classical CGHS solution in the gauge $\rho=\phi$ [8]. Solving the constraints gives

$$
\tilde{\phi}=a+b x+\lambda^{2} x^{2}-\frac{1}{4} \int d x \int d x\left(\pi_{f}^{2}+\left(f^{\prime}\right)^{2}\right) \quad, \quad \pi_{\tilde{\rho}}=c+\frac{1}{2} \int d x \pi_{f} f^{\prime},
$$

so that the independent canonical variables (or true degrees of freedom) are $\left(\pi_{f}, f\right)$ canonical pairs. The reduced phase space Hamiltonian is a free-field Hamiltonian

$$
H=\frac{1}{2} \int_{-\infty}^{\infty} d x\left(\pi_{f}^{2}+\left(f^{\prime}\right)^{2}\right)
$$

The dilaton and the original metric can be expressed in the gauge (6) as

$$
e^{-\phi}=-\lambda^{2} x^{+} x^{-}-F_{+}-F_{-} \quad, \quad d s^{2}=-e^{\phi} d x^{+} d x^{-},
$$

where

$$
F_{ \pm}=a_{ \pm}+b_{ \pm} x^{ \pm}+\int^{x^{ \pm}} d y \int^{y} d z T_{ \pm \pm}(z)
$$

The independent integration constants are $a_{+}+a_{-}$and $b_{ \pm}$, and $T_{ \pm \pm}$is the matter energy-momentum tensor

$$
T_{ \pm \pm}=\frac{1}{2} \partial_{ \pm} f \partial_{ \pm} f
$$

An equivalent form of the solution (2.10), which is suitable for our purposes, is given by

$$
F_{ \pm}=\alpha_{ \pm}+\beta_{ \pm} x^{ \pm}+\int_{\Lambda^{ \pm}}^{x^{ \pm}} d y\left(x^{ \pm}-y\right) T_{ \pm \pm}(y)
$$

The formulas (2.9-10) can be derived from the eq. (2.7) by using $\pi_{f}=\dot{f}$. 


\section{Quantum theory}

Quantum theory is defined by choosing a representation of the quantum canonical commutation relations

$$
\left[\pi_{f}(x), f(y)\right]=-i \delta(x-y)
$$

We take the standard Fock space representation, by defining the creation and anhilation operators $a^{\dagger}, a$ as

$$
a_{k}=\frac{-i \pi_{k}+k \operatorname{sign}(k) f_{k}}{\sqrt{2|k|}}
$$

where

$$
f(x)=\int_{-\infty}^{\infty} \frac{d k}{\sqrt{2 \pi}} e^{i k x} f_{k} \quad, \quad \pi_{f}(x)=\int_{-\infty}^{\infty} \frac{d k}{\sqrt{2 \pi}} e^{i k x} \pi_{k}
$$

so that eq. (3.1) is equivalent to

$$
\left[a_{k}, a_{q}^{\dagger}\right]=\delta(k-q)
$$

The Fock space $\mathcal{F}\left(a_{k}\right)$ with the vacuum $|0\rangle$ is the physical Hilbert space of the theory. The Hamiltonian (2.8) can be promoted into a Hermitian operator acting on $\mathcal{F}$ as

$$
H=\int_{-\infty}^{\infty} d k \omega_{k} a_{k}^{\dagger} a_{k}+E_{0}
$$

where $\omega_{k}=|k|$ and $E_{0}$ is the vacuum energy. Therefore one has a unitary evolution described by a Schrödinger equation

$$
i \frac{\partial}{\partial t} \Psi(t)=H \Psi(t)
$$

where $\Psi(t)$ is a normalisable state from $\mathcal{F}$. It will be convenient to work in the Heisenberg picture

$$
\Psi_{0}=e^{i H t} \Psi(t) \quad, \quad A(t)=e^{i H t} A e^{-i H t},
$$

so that

$$
f(t, x)=e^{i H t} f(x) e^{-i H t}=\frac{1}{\sqrt{2 \pi}} \int_{-\infty}^{\infty} \frac{d k}{\sqrt{2 \omega_{k}}}\left[a_{k} e^{i\left(k x-\omega_{k} t\right)}+a_{k}^{\dagger} e^{-i\left(k x-\omega_{k} t\right)}\right] .
$$

It is also useful to split eq. (3.8) into left and right moving parts, so that $f=f_{+}+f_{-}$ where

$$
f_{ \pm}\left(x^{ \pm}\right)=\frac{1}{\sqrt{2 \pi}} \int_{0}^{\infty} \frac{d k}{\sqrt{2 \omega_{k}}}\left[a_{ \pm}(k) e^{-i k x^{ \pm}}+a_{ \pm}(k)^{\dagger} e^{i k x^{ \pm}}\right]
$$

The metric is given by the operator $e^{\phi}$, which can be defined as the inverse of the $e^{-\phi}$ operator. The $e^{-\phi}$ operator in the Heisenberg picture can be easily defined 
from the expressions (2.9) and (2.10), where now $f$ is the operator given by eq. (3.8), while in the expressions for $T_{ \pm \pm}$there will be a normal ordering with respect to some vacuum in $\mathcal{F}$, which can be different from $|0\rangle$, as was the case in $[8]$. However, in this paper we are going to explore the case of Kruskal vacuum normal ordering.

Given a physical state $\Psi_{0}$, one can associate an effective metric to $\Psi(t)$ as

$$
e^{\rho_{e f f}(t, x)}=\left\langle\Psi(t)\left|e^{\phi(x)}\right| \Psi(t)\right\rangle=\left\langle\Psi_{0}\left|e^{\phi(t, x)}\right| \Psi_{0}\right\rangle \quad .
$$

Note that $e^{\rho_{e f f}}$ can be interpreted as a metric only if the quantum fluctuations are small. A criterion for this would be

$$
\sqrt{\left|\left\langle e^{2 \phi}\right\rangle-\left\langle e^{\phi}\right\rangle^{2}\right|}<\left\langle\left\langle e^{\phi}\right\rangle\right.
$$

Note that the dispersion in (3.11) cannot be zero, since otherwise it would mean that $\Psi_{0}$ is an eigenvalue of the metric. This would imply that $\Psi_{0}$ is not a normalisable state, since the spectrum of the metric is continious.

In order to calculate $e^{\rho_{e f f}}$, we use the following formal identity

$$
\left(-\lambda^{2} x^{+} x^{-}-F\right)^{-1}=e^{\phi_{0}}\left(1-e^{\phi_{0}} \delta F\right)^{-1}=e^{\phi_{0}} \sum_{n=0}^{\infty} e^{n \phi_{0}} \delta F^{n}
$$

where $F_{0}$ is a c-number function, $e^{-\phi_{0}}=-\lambda^{2} x^{+} x^{-}-F_{0}$ and $\delta F=F-F_{0}$. Then

$$
\left\langle\left(-\lambda^{2} x^{+} x^{-}-F\right)^{-1}\right\rangle=e^{\phi_{0}} \sum_{n=0}^{\infty} e^{n \phi_{0}}\left\langle\delta F^{n}\right\rangle .
$$

Note that the expression (3.13) gets slightly simplified if one choses

$$
F_{0}=\left\langle F_{+}\right\rangle+\left\langle F_{-}\right\rangle
$$

since then the $n=1$ term vanishes, and the lowest order metric gives a one loop semiclassical metric

$$
e^{-\phi_{0}}=-\lambda^{2} x^{+} x^{-}-\left\langle F_{+}\right\rangle-\left\langle F_{-}\right\rangle
$$

We now want to choose $\Psi_{0}$ such that it is as close as possible to the classical matter distribution $f_{0}\left(x^{+}\right)$describing a left-moving pulse of matter. The corresponding classical metric is described by

$$
e^{-\rho}=\frac{M\left(x^{+}\right)}{\lambda}-\lambda^{2} x^{+} \Delta\left(x^{+}\right)-\lambda^{2} x^{+} x^{-}
$$

where

$$
M\left(x^{+}\right)=\lambda \int_{-\infty}^{x^{+}} d y y T_{++}^{0}(y) \quad, \quad \lambda^{2} \Delta=\int_{-\infty}^{x^{+}} d y T_{++}^{0}(y)
$$


and $T_{++}^{0}=\frac{1}{2} \partial_{+} f_{0} \partial_{+} f_{0}$. The geometry is that of the black hole of the mass

$$
M=\lim _{x^{+} \rightarrow+\infty} M\left(x^{+}\right)
$$

and the horizon is at

$$
x^{-}=-\Delta=-\lim _{x^{+} \rightarrow+\infty} \Delta\left(x^{+}\right)
$$

The asymptotically flat coordinates $\left(\eta^{+}, \eta^{-}\right)$at the past null infinity are given by [14]

$$
\lambda x^{+}=e^{\lambda \eta^{+}}, \lambda x^{-}=-e^{-\lambda \eta^{-}},
$$

while the asymptotically flat coordinates $\left(\sigma^{+}, \sigma^{-}\right)$at the future null infinity satisfy

$$
\lambda x^{+}=e^{\lambda \sigma^{+}}, \lambda\left(x^{-}+\Delta\right)=-e^{-\lambda \sigma^{-}} .
$$

Note that a change of coordinates defines a new set of creation and anhilation operators through

$$
f_{+}=\frac{1}{\sqrt{2 \pi}} \int_{0}^{\infty} \frac{d k}{\sqrt{2 \omega_{k}}}\left[b(k) e^{-i k \eta^{+}}+b(k)^{\dagger} e^{i k \eta^{+}}\right],
$$

and similarly for the right-moving sector. The old and the new creation and anhilation operators are related by the Bogoluibov transformations [14]. However, one has to keep in mind that in our approach there is no background metric, so that the coordinates (3.20) and (3.21) could not be interpreted as the "in" and the "out" coordinates. Only in the regions of the spacetime where the relation (3.11) is satisfied, one can have a background geometry. Moreover, this background geometry can be close to the classical geometry (3.16) for early times $[8]$, so that the coordinates $(3.20)$ can be still interpreted as the "in" coordinates.

Hence we take for $\Psi_{0}$ a coherent state

$$
\Psi_{0}=e^{A}\left|0_{\eta}^{+}\right\rangle \otimes\left|0_{\eta}^{-}\right\rangle
$$

where $\left|0_{\eta}\right\rangle=\left|0_{\eta}^{+}\right\rangle \otimes\left|0_{\eta}^{-}\right\rangle$is the vacuum for the coordinates (3.20), while

$$
A=\int_{0}^{\infty} d k\left[f_{0}(k) a_{k}^{\dagger}-f_{0}^{*}(k) a_{k}\right]
$$

$f_{0}(k)$ are the Fourier modes of $f_{0}\left(x^{+}\right)$. Note that the operator $A$ is written as a linear combination of Kruskal coordinates creation and anhilation operators, which is different from taking a corresponding linear combination of the "in" operators, what was done in [8]. This choice may look unnatural, but it simplifies the calculations and it gives the result of Bose, Parker and Peleg. 


\section{Semiclassical metric}

Now we are ready to calculate the effective metric (3.13). The lowest order semiclassical metric will be given by the eq. (3.15). This requires calculating the expectation values of the $T_{ \pm \pm}$operators, and a useful formula is

$$
\left\langle 0_{\eta}|T(x)| 0_{\eta}\right\rangle=-\frac{1}{48 \pi}\left(\frac{\eta^{\prime \prime \prime}}{\eta^{\prime}}-\frac{3}{2}\left(\frac{\eta^{\prime \prime}}{\eta^{\prime}}\right)^{2}\right)=-\frac{1}{48 \pi} D_{x}(\eta)
$$

In the left-moving sector we have

$$
\begin{aligned}
\left\langle 0_{\eta}\left|e^{-A} T_{++} e^{A}\right| 0_{\eta}\right\rangle & =\left\langle 0_{\eta}\left|T_{++}-\left[A, T_{++}\right]+\frac{1}{2}\left[A,\left[A, T_{++}\right]\right]\right| 0_{\eta}\right\rangle \\
& =-\frac{\kappa}{4\left(x^{+}\right)^{2}}+\frac{1}{2}\left(\frac{\partial f_{0}}{\partial x^{+}}\right)^{2},
\end{aligned}
$$

where $\kappa=\frac{N}{24 \pi}$. In the right-moving sector we obtain

$$
\left\langle 0_{\eta}\left|T_{--}\right| 0_{\eta}\right\rangle=-\frac{\kappa}{4\left(x^{-}\right)^{2}}
$$

from the formula (4.1). By inserting the eqs (4.2-3) into the formula (3.15) we obtain

$$
e^{-\rho_{0}}=C+b_{ \pm} x^{ \pm}-\lambda^{2} x^{+} x^{-}-\frac{\kappa}{4} \log \left(-\lambda^{2} x^{+} x^{-}\right)-\frac{1}{2} \int_{\Lambda^{+}}^{x^{+}} d y^{+}\left(x^{+}-y^{+}\right)\left(\frac{\partial f_{0}}{\partial y^{+}}\right)^{2}
$$

which is the BPP solution. It is a solution of the equations of motion of an effective one-loop action

$$
S_{e f f}=S_{0}-\frac{N}{96 \pi} \int \sqrt{-g} R \square^{-1} R-\frac{N}{24 \pi} \int \sqrt{-g}\left(R \phi-(\nabla \phi)^{2}\right)
$$

where $S_{0}$ is the classical CGHS action [9].

By setting $b_{ \pm}=0$ and by choosing $C=\frac{1}{4} \kappa[1-\log (\kappa / 4)]$ one can obtain a consistent semiclassical geometry [9]. In the case of the shock-wave matter, one has a static geometry for $x^{+}<x_{0}^{+}$

$$
e^{-\rho_{0}}=e^{-\phi_{0}}=C-\lambda^{2} x^{+} x^{-}-\frac{\kappa}{4} \log \left(-\lambda^{2} x^{+} x^{-}\right)
$$

which is defined for $\sigma<\sigma_{c r}$, where $\sigma$ is the spatial coordinate. At $\sigma=\sigma_{c r}$ there is a singularity, and this line is interpreted as a boundary of a strong coupling region. The same phenomenon occurs for the RST metric [15], and a consistent geometry can be defined for $\sigma<\sigma_{c r}$ by imposing a refelecting boundary conditions at $\sigma=\sigma_{c r}$.

For $x^{+}>x_{0}^{+}$one obtains an evaporating black hole solution

$$
e^{-\rho_{0}}=C+\frac{M}{\lambda}-\lambda^{2} x^{+}\left(x^{-}+\Delta\right)-\frac{\kappa}{4} \log \left(-\lambda^{2} x^{+} x^{-}\right) .
$$


The corresponding Hawking radiation flux at future null-infinity is determined by evaluating

$$
\left\langle 0_{\eta}\left|T_{--}\left(\xi^{-}\right)\right| 0_{\eta}\right\rangle
$$

where $T_{--}\left(\xi^{-}\right)$is normal ordered with respect to asymptoticaly flat coordinates $\xi^{ \pm}$ at future null-infinity of the metric (4.7). The $\xi^{ \pm}$coordinates are the same as the out coordinates (3.21) of the classical black hole solution. Then by using (4.1) one obtains

$$
2 \pi\left\langle T_{--}\left(\sigma^{-}\right)\right\rangle=\frac{\lambda^{2}}{48}\left[1-\left(1+\lambda \Delta e^{\lambda \sigma^{-}}\right)^{-2}\right],
$$

which corresponds to the thermal Hawking radiation, with $T_{H}=\frac{\lambda}{2 \pi}$ [2], 14]. The Hawking radiation shrinks the apparent horizon of the solution (4.7), so that the apparent horizon line meets the curvature singularity in a finite proper time, at $\left(x_{i n t}^{+}, x_{i n t}^{-}\right)$, after which the singularity becomes naked. However, a static solution

$$
e^{-\rho_{0}}=C-\lambda^{2} x^{+}\left(x^{-}+\Delta\right)-\frac{\kappa}{4} \log \left(-\lambda^{2} x^{+}\left(x^{-}+\Delta\right)\right)
$$

can be continuously matched to (4.7) along $x^{-}=x_{\text {int }}^{-}$. A small negative energy shock-wave emanates from that point, and for $x^{-}>x_{i n t}^{-}$the Hawking radiation stops, and the static geometry (4.10) has a null ADM mass. There is again a critical line $\sigma=\sigma_{c r}$, corresponding to a singularity of the metric (4.10), which can be interpreted as the boundary of the region where higher order corrections become important. The spatial geometry of the remnant (4.10) is that of a semi-infinite throat, extending to the strong coupling region.

Given the formula (3.13) we have a way to see when the BPP geometry is a good approximation. This will happen if

$$
e^{2 \rho_{0}}\left\langle\delta F^{2}\right\rangle<<1 .
$$

This condition will generically fail for $e^{-\rho_{0}}=0$, i.e. at the singularities of the BPP metric.

\section{Second order corrections}

Calculating the $\left\langle\delta F^{n}\right\rangle$ terms will require calculating $\left\langle T\left(x_{1}\right) \cdots T\left(x_{2}\right)\right\rangle$ and this will require a regularization. As was discussed in [8], the singularity structure of such an expression is encoded in the operator product expansion

$$
T(x) T(y)=\frac{c / 2}{(x-y)^{4}}+\frac{2 T(y)}{(x-y)^{2}}+\frac{2 \partial T(y)}{x-y}+\text { const. }+o(x-y),
$$


The simplest ordering (regularization) is

$$
: T\left(x_{1}\right) \cdots T\left(x_{n}\right):=T\left(x_{1}\right) \cdots T\left(x_{n}\right)-\left\langle 0\left|T\left(x_{1}\right) \cdots T\left(x_{n}\right)\right| 0\right\rangle \quad,
$$

where $|0\rangle$ is the relevant vacuum (i.e. $\langle 0|T| 0\rangle=0$ ). However, this removes only the leading divergencies coming from the $(x-y)^{-4}$ term, and more sophisticated schemes can be employed based on the point-splitting method $[\mathbb{8}$. We apply these methods for calculating the second order corrections.

It will be usefull to redefine $F$ as

$$
\begin{aligned}
e^{-\phi} & =\alpha+\beta_{ \pm} x^{ \pm}-\lambda^{2} x^{+} x^{-}-\int_{\Lambda^{ \pm}}^{x^{ \pm}} d y(x-y)\left\langle 0_{\eta}\left|T_{ \pm \pm}(y)\right| 0_{\eta}\right\rangle \\
& -\frac{1}{2} \int_{\Lambda^{ \pm}}^{x^{ \pm}} d y^{+}\left(x^{+}-y^{+}\right)\left(T_{ \pm \pm}(y)-\left\langle 0_{\eta}\left|T_{ \pm \pm}(y)\right| 0_{\eta}\right\rangle\right) \\
& =C-\lambda^{2} x^{+} x^{-}-\frac{\kappa}{4} \log \left(-\lambda^{2} x^{+} x^{-}\right)-F_{+}-F_{-}
\end{aligned}
$$

so that new $F_{ \pm}$are given as

$$
F_{ \pm}=\int_{\Lambda^{ \pm}}^{x^{ \pm}} d y\left(x^{ \pm}-y\right) \tilde{T}_{ \pm \pm}(y)
$$

where $\tilde{T}=T-\left\langle 0_{\eta}|T| 0_{\eta}\right\rangle$ and $\left\langle 0_{\eta}|\tilde{T}| 0_{\eta}\right\rangle=0$. Another convinient redefinition is to rescale $f(x)$ to $\sqrt{2 \pi} f(x)$.

In the left sector we define

$$
: T\left(x_{1}\right) T\left(x_{2}\right):=T\left(x_{1}\right) T\left(x_{2}\right)-\left\langle 0_{\eta}\left|T\left(x_{1}\right) T\left(x_{2}\right)\right| 0_{\eta}\right\rangle \quad,
$$

so that

$$
\begin{aligned}
\left\langle 0_{\eta}\left|: \tilde{T}_{A}\left(x_{1}\right) \tilde{T}_{A}\left(x_{2}\right):\right| 0_{\eta}\right\rangle & =\left\langle 0_{\eta}\left|\left[A, T_{1}\right]\left[A, T_{2}\right]\right| 0_{\eta}\right\rangle \\
& =-\frac{1}{4 \pi^{2}} \frac{\partial f_{0}}{\partial x_{1}} \frac{\partial f_{0}}{\partial x_{2}}\left\langle 0_{\eta}\left|\frac{\partial f}{\partial x_{1}} \frac{\partial f}{\partial x_{2}}\right| 0_{\eta}\right\rangle \\
& =\frac{1}{8 \pi^{2}} \frac{\partial f_{0}}{\partial x_{1}} \frac{\partial f_{0}}{\partial x_{2}} \partial_{x_{1}} \partial_{x_{2}} \log \left|\eta\left(x_{1}\right)-\eta\left(x_{2}\right)\right| .
\end{aligned}
$$

where we have used

$$
\left\langle 0_{\eta}\left|f\left(x_{1}\right) f\left(x_{2}\right)\right| 0_{\eta}\right\rangle=-\frac{1}{2} \log \left|\eta\left(x_{1}\right)-\eta\left(x_{2}\right)\right| .
$$

We have omitted the + indicies for the simplicity sake.

The expression (5.6) is still divergent when $x_{1} \rightarrow x_{2}$ since

$$
\partial_{x_{1}} \partial_{x_{2}} \log \left|\eta\left(x_{1}\right)-\eta\left(x_{2}\right)\right|=\frac{1}{\left(x_{1}-x_{2}\right)^{2}}+\frac{1}{6} D_{x_{1}}(\eta)+o\left(x_{1}-x_{2}\right)
$$


However, one can use

$$
\int_{0}^{\infty} d k k e^{i k\left(\eta_{1}-\eta_{2}\right)}=-\frac{1}{\left(\eta_{1}-\eta_{2}\right)^{2}} \quad, \quad \eta_{1}-\eta_{2} \neq 0
$$

to rewrite (5.6) as

$$
-\frac{1}{8 \pi^{2}} \int_{0}^{\infty} d k k e^{i k\left(\eta_{1}-\eta_{2}\right)} \partial_{x_{1}} \eta \partial_{x_{2}} \eta \partial_{x_{1}} f_{0} \partial_{x_{2}} f_{0}
$$

This gives

$$
\begin{aligned}
\left\langle\delta F_{+}^{2}\right\rangle & =-\frac{1}{8 \pi^{2} \lambda^{4}} \prod_{i=1}^{2} \int_{-\infty}^{\eta^{+}} d \eta_{i}\left(e^{\lambda\left(\eta^{+}-\eta_{i}\right)}-1\right) \int_{0}^{\infty} d k k e^{i k\left(\eta_{1}-\eta_{2}\right)} \frac{\partial f_{0}}{\partial \eta_{1}} \frac{\partial f_{0}}{\partial \eta_{2}} \\
& =-\frac{1}{8 \pi^{2} \lambda^{4}} \int_{0}^{\infty} d k k\left|\mathcal{F}\left(k, \eta^{+}\right)\right|^{2},
\end{aligned}
$$

where

$$
\mathcal{F}\left(k, \eta^{+}\right)=\int_{-\infty}^{\eta^{+}} d \eta e^{i k \eta}\left(e^{\lambda\left(\eta^{+}-\eta\right)}-1\right) \frac{\partial f_{0}}{\partial \eta}
$$

Then by choosing $f_{0}$ which falls quickly enough away from the centre of the matter pulse, we can get $\mathcal{F}(k)$ such that (5.11) is finite. However, if one wants convergence for arbitrary $f_{0}$ 's of compact support, then additional regularization is needed. One way would be by subtracting $\left(x_{1}-x_{2}\right)^{-2}$ from $\partial_{1} \partial_{2} \log \left(\eta_{1}-\eta_{2}\right)$, which corresponds to using a new ordering

$$
\begin{aligned}
: T\left(x_{1}\right) T\left(x_{2}\right): & =T\left(x_{1}\right) T\left(x_{2}\right)-\left\langle 0_{\eta}\left|T\left(x_{1}\right) T\left(x_{2}\right)\right| 0_{\eta}\right\rangle \\
& -\left\langle 0_{x}\left|\left[A, T\left(x_{1}\right)\right]\left[A, T\left(x_{2}\right)\right]\right| 0_{x}\right\rangle .
\end{aligned}
$$

This gives for $\left\langle\delta F_{+}^{2}\right\rangle$

$$
\frac{1}{8 \pi^{2}} \prod_{i=1}^{2} \int_{\Lambda^{+}}^{x^{+}} d x_{i}\left(x^{+}-x_{i}\right)\left(\partial_{x_{1}} \partial_{x_{2}} \log \left|\eta^{+}\left(x_{1}\right)-\eta^{+}\left(x_{2}\right)\right|-\frac{1}{\left(x_{1}-x_{2}\right)^{2}}\right) \frac{\partial f_{0}}{\partial x_{1}} \frac{\partial f_{0}}{\partial x_{2}} \text {. }
$$

In the right sector we use the point-splitting method for regularizing the operator products. It amounts to calculating an appropriate limes $\left(x_{2 i-1} \rightarrow x_{2 i}\right)$ of the expression

$$
\left\langle 0_{\eta}\left|\prod_{i=1}^{2 n} \partial_{x_{i}^{-}} f\left(\eta\left(x_{i}\right)\right)\right| 0_{\eta}\right\rangle
$$

Expression (5.13) can be calculated by using Wick's theorem and by using the expression for a two-point function (5.7). A particular normal ordering can be chosen by an appropriate subtraction of the products of the terms $\left(x_{i}^{-}-x_{j}^{-}\right)^{-2}$ and $\partial_{k} f_{k} \cdots \partial_{l} f_{l}$ from the expression (5.13) before taking the limes, such that one obtains a regular expression after taking the limes. A useful formula for doing this is (5.8). 
For example,

$$
2 \pi T\left(x_{1}\right)=\lim _{x_{2} \rightarrow x_{1}} \frac{1}{2}\left(\frac{\partial f}{\partial x_{1}} \frac{\partial f}{\partial x_{2}}+\frac{1}{2} \frac{1}{\left(x_{1}-x_{2}\right)^{2}}\right)
$$

which gives the result (4.1). In the $n=2$ case, one can define analogously

$$
\begin{aligned}
4 \pi^{2}: T\left(x_{1}\right) T\left(x_{2}\right):= & \lim _{x_{3} \rightarrow x_{1}} \lim _{x_{4} \rightarrow x_{2}} \frac{1}{4}\left(\frac{\partial f}{\partial x_{1}} \frac{\partial f}{\partial x_{3}} \frac{\partial f}{\partial x_{2}} \frac{\partial f}{\partial x_{4}}+\frac{1}{2} \frac{1}{\left(x_{1}-x_{3}\right)^{2}} \frac{\partial f}{\partial x_{2}} \frac{\partial f}{\partial x_{4}}\right. \\
& \left.+\frac{1}{2} \frac{1}{\left(x_{2}-x_{4}\right)^{2}} \frac{\partial f}{\partial x_{1}} \frac{\partial f}{\partial x_{3}}+\frac{1}{4} \frac{1}{\left(x_{1}-x_{3}\right)^{2}} \frac{1}{\left(x_{2}-x_{4}\right)^{2}}\right)
\end{aligned}
$$

This gives

$$
\begin{aligned}
\left\langle 0_{\eta}\left|: T_{--}\left(x_{1}\right) T_{--}\left(x_{2}\right):\right| 0_{\eta}\right\rangle & =\frac{1}{32 \pi^{2}}\left(\partial_{x_{1}} \partial_{x_{2}} \log \left|\eta^{-}\left(x_{1}\right)-\eta^{-}\left(x_{2}\right)\right|\right)^{2} \\
& +\left\langle T_{--}\left(x_{1}\right)\right\rangle\left\langle T_{--}\left(x_{2}\right)\right\rangle
\end{aligned}
$$

The expression (5.17) is still divergent when $x_{1} \rightarrow x_{2}$, and it will be the source of divergence in

$$
\left\langle\delta F_{-}^{2}\right\rangle=\frac{1}{32 \pi^{2}} \int_{\Lambda^{-}}^{x^{-}} \int_{\Lambda^{-}}^{x^{-}} d x_{1} d x_{2}\left(x^{-}-x_{1}\right)\left(x^{-}-x_{2}\right)\left(\partial_{x_{1}} \partial_{x_{2}} \log \left|\eta^{-}\left(x_{1}\right)-\eta^{-}\left(x_{2}\right)\right|\right)^{2} .
$$

One way to regularize (5.18) is by changing the definition (5.16) [8]. One can define

$$
\begin{aligned}
& 4 \pi^{2}: T\left(x_{1}\right) T\left(x_{2}\right):=\lim _{x_{3} \rightarrow x_{1}} \lim _{x_{4} \rightarrow x_{2}}\left(\frac{1}{4} \frac{\partial f}{\partial x_{1}} \frac{\partial f}{\partial x_{3}} \frac{\partial f}{\partial x_{2}} \frac{\partial f}{\partial x_{4}}\right. \\
& +\frac{1}{2} \frac{1}{\left(x_{1}-x_{3}\right)^{2}} \frac{\partial f}{\partial x_{2}} \frac{\partial f}{\partial x_{4}}+\frac{1}{2} \frac{1}{\left(x_{2}-x_{4}\right)^{2}} \frac{\partial f}{\partial x_{1}} \frac{\partial f}{\partial x_{3}}+\frac{1}{4} \frac{1}{\left(x_{1}-x_{3}\right)^{2}} \frac{1}{\left(x_{2}-x_{4}\right)^{2}} \\
& +\frac{1}{2} \frac{1}{\left(x_{1}-x_{2}\right)^{2}} \frac{\partial f}{\partial x_{3}} \frac{\partial f}{\partial x_{4}}+\frac{1}{2} \frac{1}{\left(x_{3}-x_{4}\right)^{2}} \frac{\partial f}{\partial x_{1}} \frac{\partial f}{\partial x_{2}}+\frac{1}{4} \frac{1}{\left(x_{1}-x_{2}\right)^{2}} \frac{1}{\left(x_{3}-x_{4}\right)^{2}} \\
& \left.+\frac{1}{2} \frac{1}{\left(x_{1}-x_{4}\right)^{2}} \frac{\partial f}{\partial x_{2}} \frac{\partial f}{\partial x_{3}}+\frac{1}{2} \frac{1}{\left(x_{2}-x_{3}\right)^{2}} \frac{\partial f}{\partial x_{1}} \frac{\partial f}{\partial x_{4}}+\frac{1}{4} \frac{1}{\left(x_{1}-x_{4}\right)^{2}} \frac{1}{\left(x_{2}-x_{3}\right)^{2}}\right),
\end{aligned}
$$

so that

$$
\begin{aligned}
\left\langle 0_{\eta}\left|: T_{--}\left(x_{1}\right) T_{--}\left(x_{2}\right):\right| 0_{\eta}\right\rangle & =\frac{1}{32 \pi^{2}}\left(\partial_{x_{1}} \partial_{x_{2}} \log \left|\eta^{-}\left(x_{1}\right)-\eta^{-}\left(x_{2}\right)\right|-\frac{1}{\left(x_{1}-x_{2}\right)^{2}}\right)^{2} \\
& +\left\langle T_{--}\left(x_{1}\right)\right\rangle\left\langle T_{--}\left(x_{2}\right)\right\rangle
\end{aligned}
$$

(5.20) is finite for $x_{1} \rightarrow x_{2}$ due to eq. (5.8). Consequently $\left\langle\delta F_{-}^{2}\right\rangle$ is given by

$$
\frac{1}{32 \pi^{2}} \prod_{i=1}^{2} \int_{\Lambda^{-}}^{x^{-}} d x_{i}\left(x^{-}-x_{i}\right)\left(\partial_{x_{1}} \partial_{x_{2}} \log \left|\eta^{-}\left(x_{1}\right)-\eta^{-}\left(x_{2}\right)\right|-\frac{1}{\left(x_{1}-x_{2}\right)^{2}}\right)^{2},
$$

which is finite. 


\section{Conclussions}

We have shown that in a unitary gauge quantization of the CGHS model one can obtain an evaporating black hole solution in the semiclassical limit, which is also a solution of the one-loop effective action proposed by BPP, which is manifestly diffeomorphism invariant. This partially answers a dillema about the diffeomorphism invariance of our construction, since it means that the diffeomorphism invariance is preserved in the lowest order of the expansion (3.13). However, the drawback is that it is not obvious which regularization procedure will give a diffeomorphism invariant expressions for the higher order corrections $\left\langle\delta F^{n}\right\rangle,(n \geq 2)$. This problem is being investigated [16].

Also note that in the unitary gauge quantization there is no restriction on the number of matter fields $N$, and one could take $N=1$. Since the constraints of the theory can be transformed into the constraints of an $(N+2)$-dimensional bosonic string [4, 5, 7, 17], one could worry about the diffeo anomalies for $N \neq 24$, which appear in the Dirac quantization of the bosonic string. However, Kuchar and Torre have shown that an anomaly free Dirac quantization of the bosonic string is possible for any $N$ [18]. Although the Kuchar-Torre construction requires canonical variables such that the constraints become non-polynomial, and that makes them difficult to solve, it shows that it is possible to preserve the diffeo invariance for $N \neq 24$. Hence it is not surprising that in a unitary gauge quantization approach the diffeomorphism invariance is preserved at one-loop order. This also lays at rest doubts that a diffeomorphism invariant quantization of the CGHS model can give a free-field Fock space as a physical Hilbert space [19].

The quantum gravity theory we have constructed has a fixed space-time manifold $\mathbf{R}^{2}$, with differential structure (i.e. coordinates) but without metric. The wavefunctions of the theory are defined on $t=$ const. surfaces, which are related by a unitary transformation (3.7). The dilaton and the metric are induced by the matter, which propagates as a free field. The space-time geometry is given by the expectation value of the metric and the dilaton operator, and it is defined only in the regions of $\mathbf{R}^{2}$ where the quantum fluctuations are small. The one-loop results indicate that the quantum fluctuations become large at the curvature singularity, which would indicate a notion of a classical singularity as a place where the geometry becomes fuzzy.

The unitarity of the theory implies that an observer located at $x=\infty$ who sends the matter pulse to $x=-\infty$ at $t=-\infty$ will generically know the complete wavefunction at later times. However, when observer performs a measurement, the wavefunction will collapse into an eigenvalue state of his observables. If the mea- 
surement is performed before the time $t_{0}$ when the apparent horizon forms in the effective metric (3.10), the outcome of the measurement will be completely known to the observer, and it will be a state in $\mathcal{H}$. For $t>t_{0}$ the outcome of the measurement will be again a state in $\mathcal{H}$, but the observer will only know the part of the state which is associated to the observables lying outside of the horizon. Hence for $t>t_{0}$ he will have to use a density matrix in the Hilbert space $\mathcal{H}_{\text {outs }}(t)$, to describe the results of his measurements $\left(\mathcal{H}_{\text {outs }}\right.$ is the Hilbert space corresponding to the observables defined outside of the horizon). However, because of the unitarity, the relation

$$
\mathcal{H}=\mathcal{H}_{\text {ins }}(t) \otimes \mathcal{H}_{\text {outs }}(t)
$$

will be always valid $\left(\mathcal{H}_{\text {ins }}\right.$ is the Hilbert space corresponding to observables inside the horizon). Hence whether the observer will see a pure state or a mixed state will depend on the behaviour of the exact quantum metric (3.10). Given the oneloop approximation (3.15), it is still unclear what will be the effect of the higher order corrections in the strong copupling region, but since the theory is unitary, one has two known possibilities: remnants or the complete evaporation with the information being returned through the radiation. The one-loop result seems to favour the remnant scenario. However, a further work is neccessary for an answer to this question.

\section{Acknowledgements}

I would like to thank Voja Radovanović and Don Marolf for useful discussions.

\section{References}

[1] S.W. Hawking, Commun. Math. Phys.43 (1975) 199

[2] C.G. Callan, S.B. Giddings, J.A. Harvey and A. Strominger, Phys. Rev. D45 (1992) R1005

[3] A. Strominger, Les Houches Lectures on Black Holes, Santa Barbara preprint (1995), hep-th/9501071

[4] A. Miković, Phys. Lett. 291B (1992) 19

[5] S. Hirano, Y. Kazama and Y. Satoh, Phys. Rev. D48 (1994) 1687

[6] A. Miković, Phys. Lett. 304B (1993) 70 
[7] A. Miković, Black holes and nonperturbative canonical 2d dilaton gravity, Imperial College preprint Imperial-TP/95-94/16, hep-th/9402095, (1994)

[8] A. Miković, Hawking radiation and backreaction in a unitary theory of 2d quantum gravity SISSA preprint 98/94/EP, hep-th/9407104, to appear in Phys. Lett. $\mathrm{B}$

[9] S. Bose, L. Parker and Y. Peleg, Semi-infinite throat as the end-state geometry of $2 d$ black hole evaporation, Univ. of Winsconsin-Milwaukee preprint WISCMILW-95-TH-10 (1995)

[10] B.K. Berger, D.M. Chitre, V.E. Moncrief and Y. Nutku, Phys. Rev. D5 (1972) 2467

[11] W.G. Unruh, Phys. Rev. D14 (1976) 870

[12] P. Hajicek, Phys. Rev. D30 (1984) 1178

[13] A. Bilal and I. Kogan, Phys. Rev. D47 (1993) 5408

[14] S.B. Giddings and W.M. Nelson, Phys. Rev. D46 (1992) 2486

[15] J.G. Russo, L. Susskind and L. Thorlacius, Phys. Rev. D46 (1992) 3444; Phys. Rev. D47 (1993) 533

[16] A. Miković and V. Radovanović, work in progress

[17] D. Cangemi and R. Jackiw, Phys. Lett. 337B (1994) 271

[18] K. Kuchar and G. Torre, J. Math. Phys 30 (1989) 1769

K. Kuchar, Phys. Rev. D39 (1989) 2263

[19] D.Cangemi, R. Jackiw and B. Zwiebach, MIT preprint (1995) 